\title{
Origin and Evolutionary History of HIV-1 Subtype B in Cuba
}

\author{
Liuber Y. Machado-Zaldívar MS PhD, Héctor M. Díaz-Torres MD MS PhD, Madeline Blanco-de Armas PhD, \\ Dania Romay-Franchy, Marta Dubed-Echevarría MS
}

\begin{abstract}
INTRODUCTION Cuba's HIV epidemic is characterized by high genetic diversity, with circulation of several subtypes and recombinant forms. Earlier studies described a predominance of subtype B in the HIV-positive population, but these studies did not take into account patients' epidemiologic history.
\end{abstract}

OBJECTIVE Clarify the origin and phylodynamics of HIV-1 subtype B in the Cuban epidemic.

METHODS We analysed phylogenetic relationships among 120 sequences (from different geographic origins) of the pol gene in HIV-1 subtype B isolates from Cuban patients diagnosed from 1987 through 2012. Time of HIV-1 subtype B introduction and viral evolutionary rate were determined using a Bayesian coalescent method.

\section{INTRODUCTION}

HIV-1 arose in the early 20th century from cross-species transmission of simian immunodeficiency virus from chimpanzees to humans in Central and West Africa.[1] HIV-1 group M, responsible for the majority of cases worldwide, initially spread throughout Africa and in response to various genetic forces, diversified into different subtypes, among them subtype B.[2-4] Spread of these variants in the human population was not noticed until 1981, when the first patients with symptoms of AIDS were described in the USA; they were infected with HIV-1 subtype B (HIV-1B).[5,6]

Spread of subtype B from Africa to the Americas initially occurred via its introduction into Haiti in the 1960 s, probably with the return of Haitian professionals from work missions in the Democratic Republic of the Congo. Following this, it spread to the USA and from there to the rest of the world, as pandemic clade B.[7] Another subtype B lineage known as Caribbean B is found only in Haiti and neighboring Caribbean islands.[8] Because subtype $B$ was the genetic variant isolated in industrialized nations and the first to disperse with population movements, its spread is the subject of continuing debate in the field of phylogeography. $[9,10]$

The first molecular epidemiology studies done in Cuba reported circulation of several genetic variants of HIV-1, with a predominance of subtype B in the HIV-positive population (primarily men who have sex with men).[11] Later studies also described high genetic variability, with multiple circulating recombinant forms, still with a predominance of subtype B.[12-18] Several studies by nonCuban authors have analyzed the phylodynamics of Cuba's HIV1 epidemic, but have not taken into account the epidemiologic history of the patients studied.[19-21]

This study aimed to clarify the evolutionary history of HIV-1 subtype B in a sample of the HIV-positive population and to learn whether the subtype $B$ virus variants transmitted are associated with variants detected at the epidemic's outset. Another objective
RESULTS Based on phylogenetic relationships, subtype B was introduced into Cuba multiple times. Subtype B spread in Cuba through dissemination of strains that probably came from the USA, Canada and Europe. The time of the most recent common ancestor of Cuban subtype B was close to 1977 (95\% Cl 1974-1982), and the evolutionary rate was $2.7 \times 10^{-3}$ nucleotide substitutions per site per year.

CONCLUSIONS Our results suggest multiple introductions of HIV-1B into Cuba in the late 1970s, predominantly strains from North America and Europe. The results reflect the importance of maintaining, reviewing and updating molecular epidemiology of HIV-1 in Cuba, due to its rapid evolution and possible implications for the National STI/HIVIAIDS Program of Cuba's Ministry of Public Health.

KEYWORDS HIV-1, subtype B, HAART, molecular epidemiology, molecular evolution, phylodynamics, Cuba

is to determine its phylodynamic pattern in the HIV-positive population several years after HAART use began in Cuba.

\section{METHODS}

\section{Selection of HIV-1B nucleotide sequence group and multiple} sequence alignment $A$ retrospective descriptive study was done to confirm geographic relationship, time of most recent common ancestor (tMRCA) and evolutionary rate $(\mu$, nucleotide substitutions per site per year) of Cuban subtype B sequences detected in resistance surveillance studies (120 sequences). We queried the database at the US Los Alamos National Laboratory on 29 January 2013 and retrieved data on 2021 sequences from protease $(\mathrm{PR})$ and reverse transcriptase $(\mathrm{RT})$ regions of the pol gene (nucleotides 2253-3233 in strain HXB2) of HIV-1B.[22]

To ensure high-quality data, sequences that met the following criteria[19] were selected:

- Cuban subtype B sequences reported in HIV antiretroviral resistance surveillance studies in 1999 (patients diagnosed in 1987-1999) and 2009-2012 (new diagnoses);

- Sequences representative of different geographic regions that include almost all continents (North America is overrepresented due to the greater availability of sequences) (Table 1);

- Sequences with no evidence of intersubtype recombination (The Recombinant Identification Program tool of the US Los Alamos database was used,[22] with a minimum confidence interval for pure subtype B of 0.95 and a window size of 200 nucleotides); and

- Sequences with no evidence of hypermutations, stop codons or excess of undetermined nucleotides.

These criteria were used to select 1746 sequences for analysis, which included 120 Cuban subtype B sequences studied in 1999 and 2009-2012. 
Table 1: HIV-1B pol gene sequences by geographic region

\begin{tabular}{|l|r|r|}
\hline Region & \multicolumn{1}{|c|}{$\mathbf{n}$} & \multicolumn{1}{c|}{$\%$} \\
\hline North America & 702 & 40.2 \\
\hline South America & 144 & 8.2 \\
\hline Caribbean & 105 & 6.0 \\
\hline Europe & 150 & 8.6 \\
\hline Asia & 415 & 23.8 \\
\hline Africa & 110 & 6.3 \\
\hline Cuba & 120 & 6.9 \\
\hline Total & 1746 & 100.0 \\
\hline
\end{tabular}

Sequences were aligned using the Clustal $X$ tool of Bioedit Sequence Alignment Editor[23] and were manually edited. Two subtype $C$ sequences were used as outgroups.

Phylogenetic analysis and reconstruction of evolutionary and demographic history To determine the geographic relationship of Cuban HIV-1B sequences to other strains from other regions, several phylogenetic trees were constructed using genetic distance, maximum likelihood and Bayesian inference methods. The tree with the greatest reproducibility and consistency was chosen, which ended up being the one constructed with the

Figure 1: Phylogenetic tree of 1746 sequences of the HIV-1B pol gene

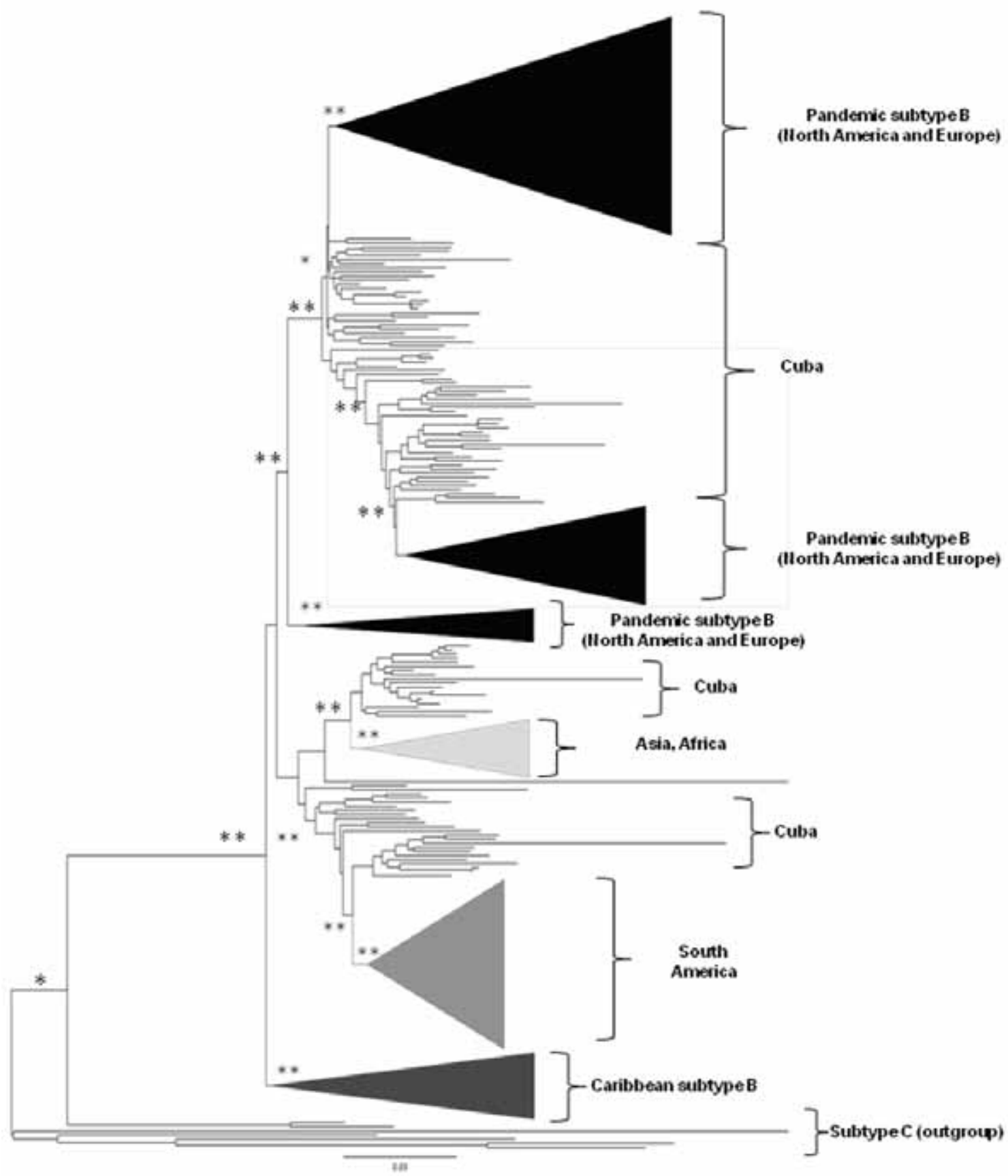

*bootstrap value $>0.80 \quad$ **bootstrap value $>0.90$

Tree constructed using neighbor-joining; genetic distance estimated by calculating matrices according to Kimura's two-parameter method. Sequences from other geographic regions were grouped and are represented with different tones. 
neighbor-joining similarity method and estimation of genetic distance based on Kimura's two-parameter method.[24] Bootstrap values were calculated using 1000 replicates, with MEGA v 5.0.[25]

A BEAST program input file was generated using the BEAUti tool from BEAST v. 1.7.4.[26] Evolutionary rate and tMRCA were estimated according to the best demographic model. The best coalescent model was determined by estimating log likelihood with the Akaike information criterion through Markov chain Monte Carlo (AICM). Several parametric coalescent demographic models were used (constant population size and exponential growth) and a nonparametric one, the Bayesian skyline plot, which were compared under strict and relaxed molecular clock conditions, with uncorrelated lognormal distribution (UCLD), and with uncorrelated exponential distribution (UCED).

The best coalescent model was chosen by applying the Bayes factor through log likelihood. Markov chain Monte Carlo was run for 300 million generations to obtain an effective sampling size of $>250$ (the value for which the method is considered reliable) for the parameters of interest. As recommended in the BEAST program manual, $10 \%$ of trees constructed were discarded. [26] Convergence was measured via estimation of effective sampling size after constructing $10 \%$ of the trees using Tracer v. 1.5.[26] The trees were plotted using FigTree v. 1.1.2.[27]

Ethics Ethical procedures met the standards of the Cuban Ministry of Public Health and of the Ministry of Science, Technology and the Environment, in conformity with the principles of the Helsinki Declaration.[28] The study was reviewed and approved by the Bioethics Committee of the AIDS Research Laboratory and included the majority of recently diagnosed adults reported in surveillance of transmitted HIV-1 resistance to antiretroviral drugs in Cuba. Data management procedures ensured patient confidentiality.

\section{RESULTS}

Geographic relationship, origin and evolutionary rate of Cuban HIV-1B sequences The majority of Cuban HIV-1B sequences were grouped with viruses from North America, as shown on the phylogenetic tree (Figure 1). Other sequences were related to viral variants from other geographic regions.

Table 2 displays the values obtained for the different coalescent models. The model chosen for analysis was the Bayesian skyline

Table 2: Demographic models of HIV-1B evolution

\begin{tabular}{|l|r|r|r|r|r|c|}
\hline Coalescent model & $\begin{array}{c}\text { Type of } \\
\text { molecular clock }\end{array}$ & $\begin{array}{c}\text { Log } \\
\text { likelihood }\end{array}$ & HME & sHME & AICM & BF \\
\hline Constant size & SC & -28.261 & -29.612 & -28.318 & 60.733 & -28.156 \\
\hline Exponential growth & SC & -28.257 & -29.947 & -28.377 & 62.867 & -28.182 \\
\hline BSP & SC & -28.230 & -30.439 & -28.352 & 65.918 & -28.113 \\
\hline Constant size & UCED & -28.129 & -31.834 & -28.318 & 87.595 & -28.307 \\
\hline Exponential growth & UCED & -28.099 & -31.168 & -28.325 & 81.968 & -28.310 \\
\hline BSP & UCED & -28.062 & -31.326 & -28.235 & 80.615 & -28.277 \\
\hline Constant size & UCLD & -28.119 & -31.313 & -28.248 & 74.836 & 28.167 \\
\hline Exponential growth & UCLD & -28.113 & -30.933 & -28.243 & 74.494 & -28.181 \\
\hline BSP & UCLD & -28.098 & -31.154 & -28.277 & 76.333 & -28.151 \\
\hline
\end{tabular}

AICM: Akaike information criterion through Markov chain Monte Carlo

BF: Bayes factor BSP: Bayesian skyline plot HME: harmonic mean estimator

SC: strict clock SHME: smoothed harmonic mean estimator

UCED: uncorrelated exponential distribution UCLD: uncorrelated lognormal distribution plot with a UCED strict molecular clock, because it showed the highest log-likelihood values.

The analysis estimated 1977 as the tMRCA date for Cuban sequences (95\% Cl 1974-1982) and estimated $\mu$ to be $2.73 \times 10^{-3}$ substitutions per site per year $\left(95 \% \mathrm{Cl} 2.37-3.09 \times 10^{-3}\right)$.

\section{DISCUSSION}

The HIV-1 epidemic in Cuba is characterized by elevated genetic diversity, which manifests in widespread circulation of subtypes and multiple recombinant forms.[11-18]

Although comparisons among countries and population groups and across studies are difficult, due to differences in the gene being analyzed, the methods used to align multiple sequences, the nucleotide substitution models and the degree of homogeneity of data used in the estimation,[29] the grouping of Cuban sequences with sequences of diverse geographic origins, primarily from North America and Europe, suggests that this subtype was introduced into the country through multiple events. This result concurs with early epidemiologic studies that detected subtype B in Cuba, and reported the infection source to be the USA and other countries, such as Ethiopia, Angola, the Democratic Republic of the Congo and Spain.[11] Furthermore, presence of independent groupings of Cuban sequences indicates indigenous transmission of this viral variant in the population diagnosed with HIV-1 infection during 2009-2012. In Cuba, subtype B had its founder effect in populations of men who have sex with men, facilitating its rapid dissemination[30] and making it the most common variant reported in the HIV-1 database of the US Los Alamos National Laboratory (34.4\%).[22]

Delatorre and Bello, who employed all Cuban sequences of the HIV-1 pol gene from 2001 to 2012 available in the Genbank database, stated that the bulk of subtype B infections in Cuba resulted from dissemination of a few founder viruses introduced from North America and Europe.[21] However, another study that analyzed the spread of this variant in the Western Hemisphere employed only 12 Cuban sequences obtained in 1999 by Pérez[14] and associated the origin of Cuban subtype B with viral strains from South America.[19]

Neither group of authors took into account the epidemiologic history of HIV-1 in Cuba; they relied on computational methods, mostly based on biological samples obtained after the estimated time of infection, which varies from case to case.[31] With respect to origin and dissemination of subtype B in the Western Hemisphere, Junqueira posed two epidemiologic scenarios: direct introduction of subtype B to South America, with a later secondary outbreak in the USA, and spread of subtype B from the Caribbean to North and South America.[19]

Spread of subtype B from Africa to the Americas initially occurred through the introduction of this subtype into Haiti in the 1960s, probably associated with the return of Haitian professionals from work missions in the Congo.[3,4,7,10] However, it must be taken into account that direct introduction of subtype B into the USA from the 
Caribbean generated a genetic fingerprint in the viral genome that is evident when studying appropriate genetic markers, such as the pol gene, which is why the phylogenetic patterns expected under such models could group a significant number of US sequences early in the epidemic with Caribbean strains.[19] However, phylogeographic reconstruction by Pagán and Holguín in 2013 indicated that subtype $B$ in the Caribbean could have originated in Puerto Rico and Antigua, and that this genetic variant in Puerto Rico, in turn, possibly originated in the USA.[9,20]

Our study estimated that HIV-1B appeared in Cuba in the late 1970s. This result falls within the confidence interval of tMRCA obtained by Pagán and Holguín in 2013, when they reconstructed the time and routes of dispersion of the subtype B epidemic in the Caribbean and Central America and estimated 1982 as tMRCA for Cuban subtype B (95\% Cl 1975-1985).[20]

In contrast, other authors place tMRCA for Cuban subtype B sequences at $1991(95 \% \mathrm{Cl} 1988-1994)$ and $1992(95 \% \mathrm{Cl}$ 1986-1994).[21] When analyzing viral sequences from Haiti that grouped with African sequences, Gilbert posed the hypothesis that subtype B spread in 1966 from Africa to the Americas (95\% Cl 1962-1970) and that Haiti was the epicenter of the introduction of subtype B into the USA before its global dissemination.[32] Keeping in mind that the first AIDS cases were described in patients in the USA in 1981,[33] there is a relationship between the estimated tMRCA obtained by Junqueira[7] and the natural history of the disease, given that there is a difference of 15 years from its possible origin and progression to AIDS; $5 \%-10 \%$ of patients remain asymptomatic from 10-15 years following infection and maintain a CD4+ lymphocyte count of $>500$ cells $/ \mathrm{mm}^{3}$ (patients with nonprogressive disease).[34]

In Cuba, the first cases of HIV infection were diagnosed in 1986, and the geographic origin of several viral variants was determined epidemiologically. In 1995, Rolo analyzed 58 sequences from the C2-V3 region of the HIV-1 env gene, from Cuban patients infected by HIV-1 in the USA, Ethiopia, Angola, Spain, Democratic Republic of the Congo, and Cuba. Some 41 of these sequences corresponded to subtype $\mathrm{B}$, with year of diagnosis ranging from 1986 to 1993.[11] In a later study to assess the emergence of antiretroviral drug resistance, $p o l$ gene sequences in 106 samples from Cuban patients diagnosed with HIV-1 infection from 1987 to 1999 indicated that $48.1 \%$ of samples corresponded to subtype B.[35] Our results for tMRCA are consistent with the epidemiologic history of the Cuban epidemic to date.

Delatorre and Bello estimated tMRCA at 1991 for Cuban subtype $B$, much later than the estimated origin of subtype $B$ in Haiti and the USA (1960-1970).[21] Their explanations are based on the fact that the tMRCA they obtained for Cuban subtype $B$ coincides with the economic crisis caused by the disintegration of the Soviet bloc in 1991 and the increase in tourism from North America and Europe, regions with extensive circulation of pandemic subtype B.[21] Several elements lead us to disagree with their conclusions.

First, in selecting sequences to study, Delatorre and Bello excluded those with mutations associated with antiretroviral resistance,[21] an aspect that according to several authors does not affect phylogenetic reconstruction of HIV-1, and that enables a more accurate estimate of the evolution of the infection.[36,37] Second, in selecting the best coalescent model, they ran Markov chain Monte Carlo for only 50 million generations,[21] which might interfere with obtaining an effective sampling size of $>250$, the value considered reliable for analysis.[27] Finally, they did not consider the epidemiologic history of the Cuban epidemic and overlooked the first studies that described circulation of subtype $B$ in Cuba based on samples from patients diagnosed with HIV-1 infection as early as 1986.[11,35]

Estimated evolutionary rates indicate a rapid dynamic in HIV$1 \mathrm{~B}$ viral populations. These figures may be overestimated due to the short time frames and presence of mutations that can be a common in intraspecific analysis of evolutionary rates.[38] The evolutionary rate obtained in our study is within the range described for other HIV-1B populations in the world.[20,21,39-42] This high evolutionary rate may be due to the fact that the region of the pol gene that codes for PR and RT enzymes is subject to selective forces, unsurprisingly, since these proteins are involved in functions in the viral replication cycle, such as infectivity and replication.[43] However, Pagán and Holguín also described a high degree of genetic conservation in the pol gene, which contradicts the high evolutionary rate found.[20] This coexistence may be the result of multiple mutations in a small number of sites located on the HIV-1 pol gene,[44] and the fact that, to be functional, these proteins require complete preservation of at least two-thirds of their sequences.[45,46]

\section{CONCLUSIONS}

Our results suggest multiple introductions of HIV-1B into Cuba in the late 1970s, predominantly strains from North America and Europe. These results underline the importance of maintaining, reviewing and updating the molecular epidemiology of HIV-1 in Cuba, due to the virus's rapid evolution and implications for the Cuban Ministry of Health's National STI/HIVIAIDS Program. $-1 /$ -

\section{REFERENCES}

1. Worobey M, Gemmel M, Teuwen DE, Haselkorn T, Kunstman K, Bunce M, et al. Direct evidence of extensive diversity of HIV-1 in Kinshasa by 1960. Nature. 2008 Oct 2;455(7213):661-4.

2. Sharp PM, Hahn BH. The evolution of HIV-1 and the origin of AIDS. Philos Trans R Soc Lond B Biol Sci. 2010 Aug 27;365(1552):2487-94.

3. Faria NR, Rambaut A, Suchard M, Baele G, Bedford T, Ward MJ, et al. The early spread and epidemic ignition of HIV-1 in human populations. Science. 2014 Oct 3;346(6205):56-61.

4. Tongo M, Dorfman JR, Martin DP. High degree of HIV-1 group M (HIV-1M) genetic diversity within circulating recombinant forms: insight into the early events of HIV-1M evolution. J Virol. 2015 Dec 9;90(5):2221-9.

5. Barre-Sinoussi F, Chermann JC, Rey F, Nugeyre MT, Chamaret S, Gruest T, et al. Isolation of a T-lymphotropic retrovirus from a patient at risk for AIDS. Science. 1983 May 20;220(4599):868-71.

6. Montagnier L, Chermann JC, Barr-Sinoussi F. A new human T-lymphotropic retroviruses: characterization and possible role in lymphadenophaty and acquired immunodeficiency syndromes. In: Gallo RC, Essex ME, Gross L, editors. Human T-cell leukemia/lymphoma virus: the family of human T-lymphotropic retroviruses: their role in malignancies and association with AIDS. New York: Cold Spring Harbor Laboratory Press; 1984. p. 363-79.

7. Junqueira DM, Almeida SE. HIV-1 subtype B: Traces of a pandemic. Virology. 2016 Aug;495:173-84.

8. Cabello M, Mendoza Y, Bello G. Spatiotemporal dynamics of dissemination of pandemic HIV-1 subtype B clades in the Caribbean. PLoS One. 2014 Aug 22;9(8):e106045.

9. Chalmet K, Staelens D, Blot S, Dinakis S, Pelgrom J, Plum J, et al. Epidemiological study of 
phylogentic transmission clusters in a local HIV1 epidemic reveals distinct differences between subtype B and non-B infections. BMC Infect Dis. 2010 Sep 7;10:262.

10. Magiorkibis G, Angelis K, Mamais I, Katzourakis A, Hatzakis A, Albert J, et al. The global spread of HIV-1 subtype B epidemic. Infect Genet Evol. 2016 Dec 46;169-79.

11. Rolo F, Miranda L, Wainberg M, Gu Z, Lobaina L, Noa E, et al. Envelope V3 Region Sequences of Cuban HIV-1 Isolates. J Acquir Immune Defic Syndr Hum Retrovirol. 1995 Jun 1;9(2):123-5.

12. Gómez C, Iglesias E, Perdomo W, Rolo F, Blanco $\mathrm{M}$, Lobaina $\mathrm{L}$, et al. Isolates from four different HIV type 1 clades circulating in Cuba. Identified by DNA sequence of the C2-V3 region. AIDS Res Hum Retroviruses. 2001 Jan 1;17(1):55-8.

13. Cuevas MT, Ruibal I, Villahermosa ML, Díaz $H$, Delgado E, Parga EV, et al. High HIV-1 genetic diversity in Cuba. AIDS. 2002 Aug 16;16(12):1643-53.

14. Pérez L, Thomson MM, Bleda MJ, Aragonés C, González Z, Pérez J, et al. HIV Type 1 molecular epidemiology in Cuba: high genetic diversity, frequent mosaicism, and recent expansion of BG intersubtype recombinant forms. AIDS Res Hum Retroviruses. 2006 Aug;22(8):724-33.

15. Machado LY, Blanco M, Dubed M, Díaz HM, Ruiz NM, Valdés $\mathrm{N}$, et al. HIV type 1 genetic diversity in newly diagnosed Cuban patients. AIDS Res Hum Retroviruses. 2012 Aug;28(8):956-60.

16. Machado LY, Dubed M, Díaz HM, Ruiz NM, Romay D, Valdés N, et al. Transmitted HIV type 1 drug resistance in newly diagnosed Cuban patients. AIDS Res Hum Retroviruses. 2013 Feb;29(2):411-4.

17. Kourí V, Alemán Y, Pérez L, Pérez J, Fonseca C Correa $\mathrm{C}$, et al. High frequency of antiviral drug resistance and non-B subtypes in HIV-1 patients failing antiviral therapy in Cuba. J Clin Virol. 2012 Dec;55(4):348-55.

18. Pérez L, Kourí V, Alemán $Y$, Abrahantes $Y$, Correa $C$, Aragonés $C$, et al. Antiretroviral drug resistance in HIV-1 therapy-naive patients in Cuba. Infect Genet Evol. 2013 Jun;16:144-50.

19. Junqueira DM, de Medeiros RM, Matte MC, Araújo LA, Chies JA, Ashton-Prolla P, et al. Reviewing the history of HIV-1: spread of subtype B in the Americas. PLoS One. 2011;6(11):e27489.

20. Pagán I, Holguín A. Reconstructing the timing and dispersion routes of HIV-1 subtype $B$ epidemics in the Caribbean and Central America: a phylogenetic story. PLoS One. 2013;8(7):e69218.

21. Delatorre E, Bello G. Phylodynamics of the HIV-1 epidemic in Cuba. PLoS One. 2013 Jul 9;8(7):e72448.

22. Los Alamos National Laboratory HIV-AIDS Databases [Internet]. New Mexico: Los Alamos National Laboratory; c2005-2017 [cited 2013 Jan 29]. Available from: http://www.hiv.lanl.gov

23. Hall TA. Bioedit: a user-friendly biological sequence aligment editor and analysis program for Windows 95/98/NT. Nucl Acids Symp Ser. 1999;41:95-8.

24. Kimura M. A simple method for estimating evolutionary rate of base substitutions through comparative studies of nucleotide sequences. $\mathrm{J} \mathrm{Mol}$ Evol. 1980 Dec;16(2):111-20.
25. Tamura KD, Peterson D, Peterson N, Stecher G, Nei M, Kumar S. MEGA 5: molecular evolutionary genetics analysis using maximum likelihood, evolutionary distance, and maximum parsimony methods. Mol Biol Evol. 2011 Oct:28(10):2731-9.

26. Drummond AJ, Suchard MA, Xie D, Rambaut A Bayesian phylogenetics with BEAUti and the BEAST 1.7. Mol Biol Evol. 2012 Aug;29(8):1969-73.

27. Drummond AJ, Rambaut A. BEAST: Bayesian evolutionary analysis by sampling trees. BMC Evol Biol. 2007 Nov 8; 7:214.

28. World Medical Association [Internet]. FerneyVoltaire (FR): World Medical Association; c2017. Helsinki Declaration. Ethical principles for medical research involving human subjects. 59nd General Assembly, Seul, Korea; 2008 Oct [cited 2017 Mar 22]. Available from: https://www.wma .net/policies-post/wma-declaration-of-helsinki -ethical-principles-for-medical-research-invol ving-human-subjects/

29. Grabowski MK, Redd AD. Molecular tools for studying HIV transmission in sexual networks. Curr Opin HIV AIDS. 2014 Mar;9(2):126-33.

30. Blanco M, Machado LY, Díaz HM, Ruiz N, Romay D, Silva E. HIV-1 genetic variability in Cuba and implications for transmission and clinical progression. MEDICC Rev. 2015 Oct;17(4):25-31.

31. Machuca R, Jorgensen LB, Theilade $P$, Nielsen C. Molecular investigation of transmission of Human Immunodeficiency Virus type I in a criminal case. Clin Diagn Lab Immunol. 2001 Sep;8(5):884-90.

32. Gilbert MT, Rambaut A, Wlasiuk G, Spira TJ, Pitchenik AE, et al. The emergence of HIVIAIDS in the Americas and beyond. Proc Natl Acad Sci USA. 2007 Nov 20;104(47):18566-70.

33. Center for Disease Control (CDC). Pneumocystis carinii pneumonia - Los Angeles. MMWR. 1981 Jun;30(21):250-2.

34. Buchbinder SP, Katz MH, Hessal NA, O’Malley PM, Holmberg SD. Long term HIV-1 infection without immunologic progression. AIDS. 1994 Aug;8(8):1123-8.

35. Ruibal-Brunet IJ, Cuevas MT, Díaz-Torres $\mathrm{H}$ Villahermosa ML, Noa-Romero E, Vázquez de Parga E, et al. Genotypic resistance mutations to antiretroviral drugs in HIV-1 B and non-B subtypes from Cuba. Rev Panam Salud Pública. 2001 Sep;10(3):174-80.

36. Hué S, Clewey JP, Cane PA, Pillay D. HIV-1 pol gene variation is sufficient for reconstruction of transmissions in the era of antiretroviral therapy. AIDS. 2004 Mar 26;18(5):719-28.

37. Yebra G, Holguín A, Pillay D, Hué S. Phylogenetic and demographic characterization of HIV-1 transmission in Madrid, Spain. Infect Genet Evol. 2013 Mar;14:232-9.

38. Pagán I, Holmes EC. Long-term evolution of the Luteoviridae: Time scale and mode of virus speciation. J Virol. 2010 Jun;84(12):6177-87.

39. Hué S, Pillay D, Clewley JP, Pybus OG. Genetic analysis reveals the complex structure of HIV-1 transmission within defined risk groups. Proc Nat Acad Sci USA. 2005 Mar 22;102(12):4425-9.

40. Hué S, Gifford RJ, Dunn D, Fernhill E, Pillay D; UK Collaborative Group on HIV Drug Resistance. Demonstration of sustained drug-resistant human immunodeficiency virus type 1 lineages circulating among treatment-naive individuals. J Virol. 2009 Mar;83(6):2645-54.

41. Salemi $M$, de Oliveira $T$, Ciccozzi $M$, Rezza G, Goodenow MM. High-resolution molecular epidemiology and evolutionary history of HIV-1 subtypes in Albania. PLoS One. 2008 Jan 2;3(1):e1390. DOI: 10.1371/journal. pone. 0001390 .

42. Kim GJ, Yun MR, Koo MJ, Shin BG, Lee JS, Kim $\mathrm{SS}$. Estimating the origin an evolution characteristics for Korean HIV type 1 subtype B using Bayesian phylogenetic analysis. AIDS Res Hum Retroviruses. 2012 Aug;28(8):880-4.

43. Freed EO, Mouland AJ. The cell biology of HIV-1 and other retroviruses. Retrovirology. $2006 \mathrm{Nov}$ $3 ; 3: 77$.

44. Nei M, Kumar S. Molecular Evolution and Phylogenetics. Oxford: Oxford University Press; 2000.

45. Ceccherini-Silberstein F, Gago F, Santoro M, Gori C, Svicher V, Rodríguez-Barrios F, et al. High sequence conservation of human immunodeficiency virus type 1 reverse transcriptase under drug pressure despite the continuous appearance of mutations. J Virol. 2005 Aug;79(16):10718-9.

46. Santos AFA, Lenbruger RB, Soares EA, Jere A, Sprinz E, Martínez AM, et al. Conservation patterns of HIV-1 RT connection and Rnase $\mathrm{H}$ domains: Identification of new mutations in NRTI-treated patients. PLoS One. 2008 Mar 12;3(3):e1781. DOI: 10.1371/journal. pone.0001781.

\section{THE AUTHORS}

Liuber Y. Machado-Zaldívar (Corresponding author: liuberyans@infomed.sld.cu), biochemist with a master's degree in molecular biology and doctorate in health sciences. Associate researcher, AIDS Research Laboratory, (LISIDA), Mayabeque, Cuba.

Héctor M. Díaz-Torres, internist with a master's degree in infectious diseases and doctorate in medical sciences. Senior researcher, Hermanos Ameijeiras Clinical-Surgical Teaching Hospital, Havana, and LISIDA, Mayabeque, Cuba.

Madeline Blanco-de Armas, biochemist with a doctorate in health sciences. Senior researcher, LISIDA, Mayabeque, Cuba.

Dania Romay-Franchy, molecular biology technician, LISIDA, Mayabeque, Cuba.

Marta Dubed-Echevarría, biologist with a master's degree in virology. Associate researcher and deputy director, LISIDA, Mayabeque, Cuba.

Submitted: March 22, 2017

Approved for publication: May 17, 2017 Disclosures: None 